\title{
POBLACIÓN Y ESTRUCTURA URBANA: APROXIMACIÓN A LA DIFERENCIACIÓN DEMOGRÁFICA DE LA CIUDAD DE SEGOVIA
}

\author{
Luis Carlos Martínez Fernández y José Antonio Luengo Gallego \\ Departamento de Geografía \\ Universidad de Valladolid
}

\section{RESUMEN}

En un contexto de atonía demográfica, el proceso de transformación urbana reciente de Segovia, a lo largo de los últimos años, aparece condicionado por los cambios habidos en el modelo de distribución de la población. Al progresivo abandono del centro, que ha dejado de desempeñar unas funciones residenciales preeminentes, se une el de los barrios obreros surgidos al socaire del desarrollo industrial. Frente al vaciamiento de estos sectores, cuyo menguante vecindario también es el que presenta las estructuras poblacionales más envejecidas, la ciudad se ha ido expandiendo al tiempo que ha ido aumentando el número de habitantes de los más recientes y alejados polígonos y urbanizaciones.

Palabras clave: Dinámica demográfica, estructura urbana, Segovia, movilidad residencial, envejecimiento poblacional.

\begin{abstract}
Population and urban structure: approach to the demografic differentiation of the city of Segovia. In a demografic weakness context, the process of recent urban transformation of Segovia, throughout the last years appears conditionated by the changes that have occurred in the model of population distribution. At the time when the city centre is abandoned, which has lost its preeminent residencial functions, working-class districts, arisen by industrial development, have been abandoned too. In contrast with these places where population average has an old age, the city has been expanded with the increase of inhabitants in the newest and farest urbanizations and housing estates.
\end{abstract}

Key words: Demografic dynamics, urban structure, Segovia, residential mobility, population aging.

Fecha de recepción: 8 de septiembre de 2004. Fecha de aceptación: 21 de febrero de 2005 . 


\section{La estructura urbana de segovia y su evolución al compás de las dinámicas demo- gráficas de los dos últimos tercios del siglo $\mathrm{XX}$}

La estructura urbana actual de Segovia (Figura 1) aparece definida a partir de un «centro», que puede ser identificado con la ciudad histórica. En ella, se diferenciaban claramente una serie de espacios más o menos contiguos: el Centro-Intramuros, verdadero elemento original, dentro del recinto amurallado, el Centro-Extramuros, en continuidad al anterior, evidenciando la primera expansión (burguesa) superficial de la ciudad, y los arrabales tradicionales de San Millán y de San Lorenzo, ejemplar núcleo de extrarradio, al quedar mucho más alejado (Martínez de Pisón, 1976). Esta configuración, que se mantuvo a lo largo del tiempo, sufrió una gran transformación desde el momento en el que la ciudad comenzó a experimentar un incremento sustancial de población, a partir del segundo tercio del siglo XX (Tabla 1). Fue entonces cuando comenzaron las ocupaciones progresivas y la edificación de nuevos terrenos, cada vez más alejados, destinados a satisfacer la creciente demanda residencial ${ }^{1}$.

Tabla 1

EVOLUCIÓN DE LA POBLACIÓN DE LA CIUDAD DE SEGOVIA ENTRE 1900 Y 2001

\begin{tabular}{|l|c|c|c|c|c|c|c|c|c|c|c|}
\hline & $\mathbf{1 9 0 0}$ & $\mathbf{1 9 1 0}$ & $\mathbf{1 9 2 0}$ & $\mathbf{1 9 3 0}$ & $\mathbf{1 9 4 0}$ & $\mathbf{1 9 5 0}$ & $\mathbf{1 9 6 0}$ & $\mathbf{1 9 7 0}$ & $\mathbf{1 9 8 1}$ & $\mathbf{1 9 9 1}$ & $\mathbf{2 0 0 1}$ \\
\hline Segovia & 14.547 & 14.910 & 16.013 & 18.027 & 24.977 & 29.568 & 33.360 & 41.880 & 53.237 & 54.375 & 54.368 \\
\hline
\end{tabular}

Fuente: I. N. E. Censo de población.

Barrios como los de La Albuera, Estación, San José-Mirasierra y Cristo del Mercado fueron surgiendo y desarrollándose al compás del continuo aumento poblacional de la ciudad. Crecimiento bien sensible entre 1930 y 1980 (35.210 habitantes), y muy especialmente en las décadas de los sesenta y setenta (8.520 y 11.357 habitantes, respectivamente).Todos ellos, sin excepción, desempeñaron la función que les fue asignada en el primer Plan General de Ordenación (1953), la de ser los «órganos residenciales de la mano de obra inmigrante» (Sesma, 1987: 75). Destacando, en este sentido, La Albuera, en donde se concentró el desarrollo de la ciudad entre 1940 y 1974, con una implicación decisiva de la Obra Sindical del Hogar, San José-Mirasierra y el propio Centro-Extramuros.

La progresiva configuración de la trama urbana segoviana desde el segundo tercio del siglo XX contribuyó definitivamente a otorgar al Centro-Extramuros y al arrabal de San Millán una clara vocación «nodal», mientras que el arrabal de San Lorenzo así como los barrios obreros aparecieron más alejados, al tiempo que su desarrollo, en diferentes fases, favoreció que el conjunto urbano adquiriese un «aspecto polinuclear» (Sesma, 1987: 52). Posteriormente, en los años setenta y comienzos de los ochenta, se procedería a la edificación, de la mano de la iniciativa privada, de los espacios libres, propiciando la densificación constructiva y el pleno desarrollo urbanístico, otorgando a la ciudad el carácter compacto que la define desde esos momentos «con una clara estructura reticular alargada, siguiendo la dirección natural de desarrollo hacia el sureste entorno al interfluvio Eresma-Clamores» (Villar, 1989: 168).

1 «La expansión constructora de la ciudad comenzó seriamente a partir de 1940. En esta fecha Segovia tenía 6.000 viviendas para una población de 24.977 habitantes, mientras que dos décadas después (1960), su número ascendía a 8.450. Ello supuso un incremento de inmuebles del orden del $40 \%$ y un crecimiento poblacional del 26\%» (VV. A.A., 1994). 


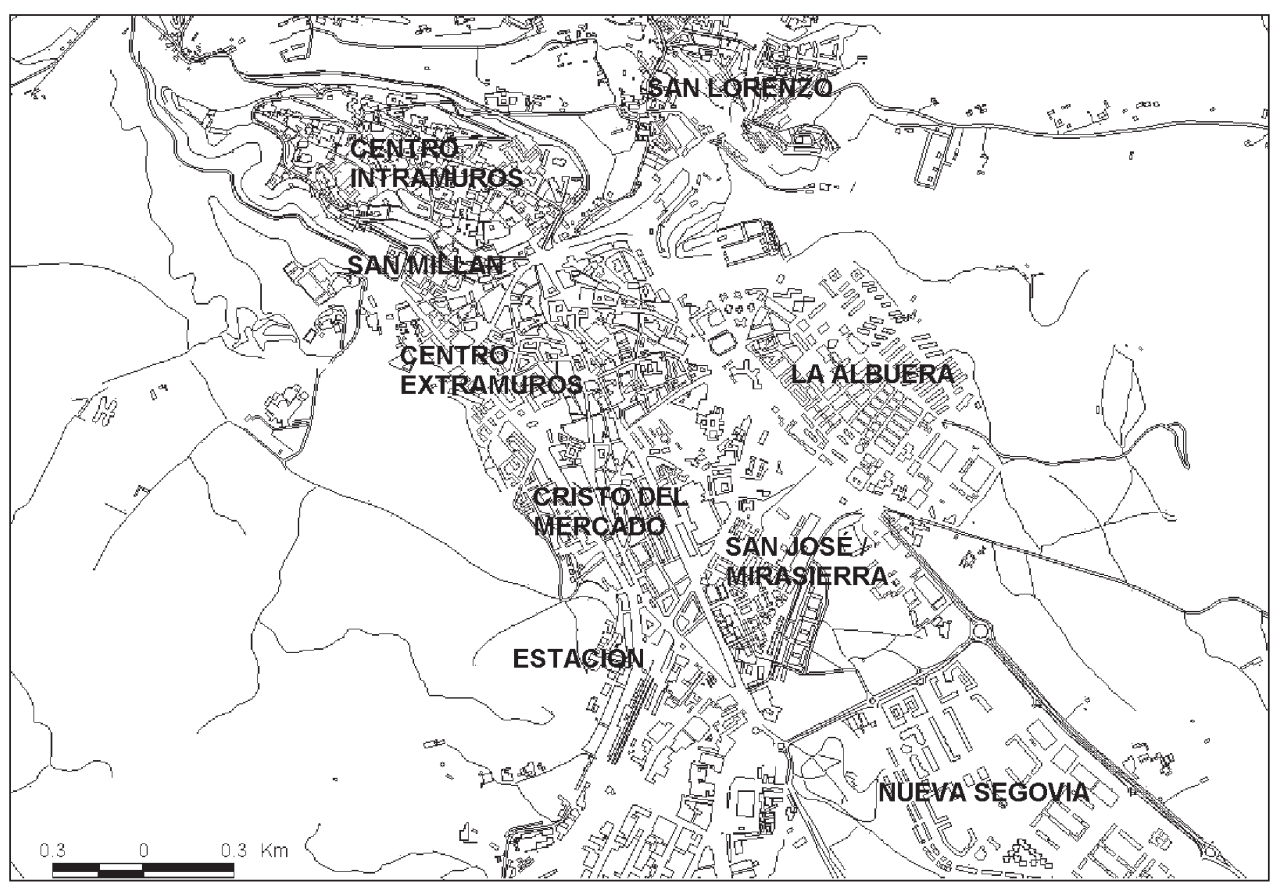

FIGURA 1. Estructura urbana de Segovia.

La planificación general de 1984 asumió plenamente la dinámica y estructura urbana anterior contemplando también la consolidación e incorporación total de un nuevo polígono residencial en la periferia de la ciudad, denominado, muy expresivamente, Nueva Segovia, lo que modificó sensiblemente el perímetro tradicional de la ciudad, haciéndolo más cuadrangular y prolongándolo en dirección a la carretera de La Granja (García y Bosque, 1989: 35). El Plan Parcial originario es de 1976 pero tuvo que ser reformulado por contener previsiones que con el transcurso de los años se manifestaron totalmente exageradas. La modificación final aprobada data de 1992 y en ella se contempla un número menor de viviendas, mucho más ajustado a la demanda real de una población que entre 1981 y 2001 apenas ha crecido en 1.000 habitantes (e incluso ha descendido según los últimos datos intercensales). Con todo, y a pesar de que las cifras se encuentran muy alejadas de las previsiones efectuadas en los setenta, Nueva Segovia se ha acabado consolidando como el nuevo espacio residencial de la capital (Luengo, 2003: 204-205).

\section{Desarrollo urbano reciente y características demográficas de los distintos barrios de la ciudad de Segovia}

En el actual marco de atonía demográfica, el crecimiento de Nueva Segovia, como el de otros espacios de la periferia, como el arrabal de San Lorenzo, solamente puede ser explicado por el paulatino y paralelo descenso del número de residentes en la ciudad compacta (tanto en el centro histórico y arrabal de San Millán como en los barrios industriales y obreros de las décadas del cuarenta al ochenta). Aparece, de este modo, un nuevo proceso de desarrollo urbano, sin apenas incrementos de población, cuyo soporte estratégico se funda- 
menta en la dialéctica entre el centro y la periferia (Calderón, 2001: 592-597). Bajo estas pautas, que caracterizan a Segovia durante el último decenio, se esconden comportamientos muy variables entre los distintos sectores espaciales que la componen, tanto desde el punto de vista de su evolución y cuantía poblacional como de sus principales estructuras, en particular las etáreas.

\subsection{La dialéctica centro-periferia en la explicación de la evolución de la población por barrios: la impronta de la movilidad residencial intraurbana}

La limitada capacidad de crecimiento demográfico de Segovia, 1.096 altas padronales entre 1996 y 2003, coexiste con una serie de fenómenos urbanísticos contradictorios, a través de los cuales se materializan los mecanismos básicos de intervención y creación de ciudad en la actualidad. El primero de estos procesos, no muy dilatado en el tiempo, pero de una notable intensidad, ha sido el progresivo abandono y vaciamiento del centro histórico y del arrabal tradicional de San Millán, que están dejando de ser los ámbitos de mayor atractivo residencial, como lo atestigua la merma de sus vecindarios (Tabla 2).

Tabla 2

EVOLUCIÓN DE LA POBLACIÓN RESIDENTE EN LA CIUDAD HISTÓRICA 1996-2003

\begin{tabular}{|l|c|c|c|}
\hline & $\mathbf{1 9 9 6}$ & $\mathbf{2 0 0 3}$ & Variación (\%) \\
\hline Centro-Extramuros & 9.870 & 9.488 & $-382(-3,87)$ \\
Centro-Intramuros & 4.841 & 4.380 & $-461(-9,52)$ \\
San Millán & 3.030 & 2.629 & $-401(-13,23)$ \\
Total ciudad ha & 17.741 & 16.497 & $-1.244(-7,01)$ \\
Total Segovia & 55.748 & 56.844 & $1.096(1,97)$ \\
\hline
\end{tabular}

Fuente: O. S.S.: Datos socioeconómicos por barrios, 1996 y 2003.

Si en 1996 vivían en el conjunto del entramado urbano histórico 17.741 personas, siete años más tarde, en 2003, lo hacían 16.497, lo que significa un decrecimiento de 1.244 habitantes, en cifras absolutas, y del 7,01\%, en términos proporcionales. Por sectores, el CentroExtramuros continua siendo el espacio más ocupado de la ciudad, con 9.488 residentes, matizándose en él la misma tendencia a la baja (382 vecinos, un 3,87\% inferior). Más importantes han sido las pérdidas de población en el Centro-Intramuros y el arrabal de San Millán. En tanto que en el primero, con un vecindario de 4.380 habitantes, éstas han ascendido a 461, representando un descenso porcentual del 9,52\%, en el segundo los 401 residentes menos suponen la mayor merma en términos relativos, ya que sus 2.629 moradores, son un $13,23 \%$ inferiores a los existentes con anterioridad.

La creciente terciarización y recualificación funcional del centro histórico (Álvarez, 1999), cada vez más impregnado de una cultura museística que pretende hacer de él una ciudad-patrimonio para el turista, la total ausencia de una adecuada política pública de recuperación del tejido edificado, que pudiera paliar el estado lamentable en el que se encuentran muchos de los inmuebles, y la carencia de inversión privada, que hace que las escasas viviendas rehabilitadas alcancen precios astronómicos, acaban erigiéndose como los factores explicativos de la pérdida de importancia residencial del «centro». Un hecho más que evidente si se tiene en cuenta que a lo largo del periodo analizado la ciudad histórica ha pasado de concentrar el $31,82 \%$ al 29,02\% de la población segoviana. 
Otro tanto ocurre en los populares barrios desarrollados al compás de los fuertes crecimientos demográficos de las décadas centrales del siglo XX. En ellos, las causas del abandono deben buscarse en factores como la insuficiencia de infraestructuras y equipamientos dotacionales o la mala calidad de las viviendas, ubicadas por lo general en edificios que han cumplido un ciclo «vital» de 45-60 años. La salida del domicilio paterno, la mejora del nivel adquisitivo y la escasa integración social-vecinal acaban por explicar la búsqueda de nuevos espacios donde vivir. Aún así, estos barrios obreros todavía aglutinan el 45,88\% de los habitantes de la ciudad, si bien esta cifra es 2,29 puntos inferior a la registrada en 1996 (Tabla 3).

Tabla 3

EVOLUCIÓN DE LA POBLACIÓN RESIDENTE EN LOS BARRIOS OBREROS 1996-2003

\begin{tabular}{|l|c|c|c|}
\hline & $\mathbf{1 9 9 6}$ & $\mathbf{2 0 0 3}$ & Variación (\%) \\
\hline Cristo del Mercado & 9.569 & 9.404 & $-165(-1,72)$ \\
La Albuera & 9.104 & 8.888 & $-216(-2,37)$ \\
Estación & 4.116 & 4.272 & $156(3,79)$ \\
San José-Mirasierra & 4.068 & 3.516 & $-552(-13,57)$ \\
Total barrios obr. & 26.857 & 26.080 & $-777(-2,90)$ \\
Total Segovia & 55.748 & 56.844 & $1.096(1,97)$ \\
\hline
\end{tabular}

Fuente: O. S.S.: Datos socioeconómicos por barrios, 1996 y 2003.

Entonces residían en la totalidad de los barrios obreros 777 personas más que en la actualidad (26.080), con lo que el decrecimiento a lo largo de estos años ha sido del $2,90 \%$, más contenido que el registrado en la ciudad histórica. De ello participan los barrios de Cristo del Mercado, La Albuera y San José-Mirasierra, aunque de una manera muy desigual. Si los dos primeros, que continúan siendo los más ocupados, 9.404 y 8.888 habitantes, han perdido población (165 y 216) por debajo de la media porcentual del conjunto $(1,72 \%$ y $2,37 \%)$, no ocurre lo mismo en San José-Mirasierra, cuyo vecindario (3.516) se ha reducido en 552 residentes, lo que supone una mengua del 13,57\%, la más alta, tanto en términos absolutos como relativos de todos los sectores que configuran la ciudad. Por su parte, La Estación participa de una dinámica completamente opuesta habiendo visto incrementar sus moradores en 156 (un 3,79\%) como consecuencia, sin lugar a dudas, de las nuevas promociones de viviendas desarrolladas recientemente, que aprovechan las buenas disponibilidades de suelo en un emplazamiento cercano al de Nueva Segovia.

Frente al descenso generalizado de población de la ciudad compacta «central», 2.021 habitantes menos en el trascurso de estos siete años, en un contexto de ligero repunte demográfico de la ciudad (1.096 empadronamientos nuevos), el crecimiento urbano periférico ha sido verdaderamente espectacular. La búsqueda de espacios libres, de nuevas tipologías edificatorias o simplemente de inmuebles de calidad, en mayor cuantía y a precios más asequibles que en el centro, son las razones que se suelen esgrimir a la hora de explicar la atracción de residentes hacia este tipo de áreas, que en el caso de Segovia serían dos: Nueva Segovia y, en menor medida, San Lorenzo (Tabla 4). 
Tabla 4

EVOLUCIÓN DE LA POBLACIÓN RESIDENTE EN LA PERIFERIA 1996-2003

\begin{tabular}{|l|c|c|c|}
\hline & $\mathbf{1 9 9 6}$ & $\mathbf{2 0 0 3}$ & Variación (\%) \\
\hline San Lorenzo & 6.040 & 6.506 & $466(7,72)$ \\
Nueva Segovia & 3.216 & 5.699 & $2.483(77,21)$ \\
Total periferia & 9.256 & 12.205 & $2.949(31,86)$ \\
Total Segovia & 55.748 & 56.844 & $1.096(1,97)$ \\
\hline
\end{tabular}

Fuente: O. S.S.: Datos socioeconómicos por barrios, 1996 y 2003.

Mientras que Nueva Segovia responde claramente a este proceso de desarrollo urbano periférico al constituir un polígono de creación «ex nova» que ha aumentado su vecindario de manera constante, desde las primeras fases urbanísticas de los años ochenta (alcanzando los incrementos más elevados de toda la ciudad, 77,21\%, entre 1996 y 2003), hasta contar en el momento presente con 5.699 habitantes, San Lorenzo sólo lo hace en parte. Se trata de uno de los dos arrabales históricos de la ciudad, el más apartado de ella, que únicamente, desde poco tiempo atrás, ha sido puesto en valor urbanístico (tanto por nueva construcción de vivienda como por rehabilitación y acondicionamiento de la ya existente). Ello explica su crecimiento en 466 personas $(7,72 \%)$ para llegar a las 6.506 residentes. En cualquier caso, en ambos espacios alejados de la ciudad tradicional y obrera viven 12.205 segovianos, 2.949 más que en 1996.

Unos crecimientos urbanos periféricos que comienzan a manifestarse más allá de los límites de la ciudad, alcanzando a varios núcleos de su entorno más inmediato. Así son cada vez más sensibles los trasvases de población hacia algunos de los denominados «barrios incorporados» o pedanías, perfilándose un incipiente proceso de desconcentración urbana y de periurbanización, que coexiste con la despoblación de aquellos otros peor situados y comunicados (Tabla 5). En Madrona, Hontoria y Revenga, la búsqueda de entornos en apariencia más saludables y tranquilos ha hecho proliferar nuevas urbanizaciones de adosados y unifamiliares a las que se han ido a vivir un buen número de residentes (114, 68 y 60, respectivamente).

Tabla 5

EVOLUCIÓN DE LA POBLACIÓN RESIDENTE EN LOS BARRIOS INCORPORADOS (PEDANÍAS) 1996-2003

\begin{tabular}{|l|c|c|c|}
\hline & $\mathbf{1 9 9 6}$ & $\mathbf{2 0 0 3}$ & Variación (\%) \\
\hline Revenga & 453 & 513 & $60(13,25)$ \\
Madrona & 376 & 490 & $114(30,32)$ \\
Zamarramala & 398 & 359 & $-39(-9,80)$ \\
Hontoria & 264 & 332 & $68(25,76)$ \\
Fuentemilanos & 307 & 294 & $-13(-4,23)$ \\
Las Serillas & 55 & 56 & $1(1,82)$ \\
Torredondo & 17 & 11 & $-6(-35,29)$ \\
Perogordo & 24 & 7 & $-17(-70,83)$ \\
Total pedanías & 1.894 & 2.062 & $168(8,87)$ \\
Total Segovia & 55.748 & 56.844 & $1.096(1,97)$ \\
\hline
\end{tabular}

Fuente: O. S.S.: Datos socioeconómicos por barrios, 1996 y 2003. 
El desarrollo de estas urbanizaciones representa la manifestación última de las nuevas condiciones de creación de ciudad en la actualidad. Ésta ya no se transforma y expande al ritmo que marcan los incrementos poblacionales, apenas 1.096 habitantes entre $1996 \mathrm{y}$ 2003, sino que lo hace en función de la dialéctica establecida entre el centro y la periferia. A través de ella, la movilidad residencial acaba erigiéndose en el factor clave a la hora de explicar las más recientes pulsaciones urbanísticas. Al abandono de la ciudad compacta (casco histórico y barrios obreros), que ha experimentado una mengua considerable en sus vecindarios (2.021 personas), se opone el crecimiento de Nueva Segovia, San Lorenzo y algunos de los «barrios incorporados», que los han aumentado en 3.117. Contrastes evidentes entre dos ámbitos espaciales que se traducen también en unas desiguales estructuras por edad, mucho más envejecidas en el primero de ellos.

\subsection{El envejecimiento como rasgo demográfico fundamental en la diferenciación espacial de la ciudad de Segovia}

La mera contemplación de las pirámides demográficas (Figuras 2, 3 y 4) muestra claramente esa realidad. A través de ellas, se percibe un doble proceso de envejecimiento, tanto por la cúspide como por la base, en la ciudad tradicional y en la práctica totalidad de los barrios obreros. Ello se debe, como se puede apreciar, con mayor nitidez, a partir de los datos estadísticos, a la escasa proporción de jóvenes y a la importancia relativa de los escalones correspondientes a la población anciana en el Centro-Intramuros (24,73\%), arrabal de San Millán $(23,89 \%)$ y Centro-Extramuros $(22,88 \%)$, así como en el conjunto de los barrios obreros: San José-Mirasierra (28,70\%), Cristo del Mercado $(20,52 \%)$ y La Albuera $(17,98 \%)$, con la única excepción de Estación $(15,03 \%)$, que también experimenta un incremento sustancial de su capacidad residencial. Frente a estos espacios, Nueva Segovia y San Lorenzo, los del relevante crecimiento periférico, presentan los vecindarios menos envejecidos, con las proporciones más altas de menores de 15 años (21,07\% y 16,88\%), que llegan a representar, en el primero de ellos, cinco veces más que la de mayores de 65 $(4,18 \%)$.

La puesta en relación de estas dos grandes cohortes etáreas (ancianos y jóvenes) viene a confirmar la diversidad espacial en el grado de envejecimiento dentro del espacio urbano de Segovia. Con un índice de 0,20, es decir, 20 personas mayores por cada cien menores de 15 años, Nueva Segovia, se desmarca claramente como el espacio menos envejecido, en clara oposición, cómo no, a los diferentes ámbitos que conforman la ciudad compacta. Barrios como los de San José-Mirasierra, San Millán y Centro-Intramuros presentan los índices más elevados $(2,41,2,28$ y 2,24), seguidos por los del Centro-Extramuros, Cristo del Mercado y La Albuera $(1,79,1,58$ y 1,28). A medio camino entre estas dos situaciones, Estación $(1,04)$ y San Lorenzo (1) evidencian su propia historia urbanística, al ser espacios en los que coexiste el antiguo vecindario envejecido de barrio obrero y de arrabal, con los más jóvenes residentes de las recientes promociones de viviendas.

Precisamente es en San Lorenzo, y en buena medida por la misma razón acentuada, donde se alcanzan los más elevados niveles de envejecimiento entre las personas mayores, con el 33,88\% de los residentes ancianos con edades superiores a los 80 años. Una excepción que confirma la regla, porque la distribución del llamado «envejecimiento del envejecimiento» permite apreciar, una vez más, los contrastes entre la ciudad histórica y obrera y los nuevos espacios periféricos. Mientras que éstos concentran los valores más bajos (Nueva Segovia, 16,39\% y Estación, 20,40\%), aquellos, dejando a un lado San Lorenzo, presentan los más altos (Centro-Intramuros, 35,36\%, Centro-Extramuros, 32,80\% y San José-Mirasierra, $27,55 \%$ ), que son superiores, en todos los casos, a los del conjunto urbano, en clara 


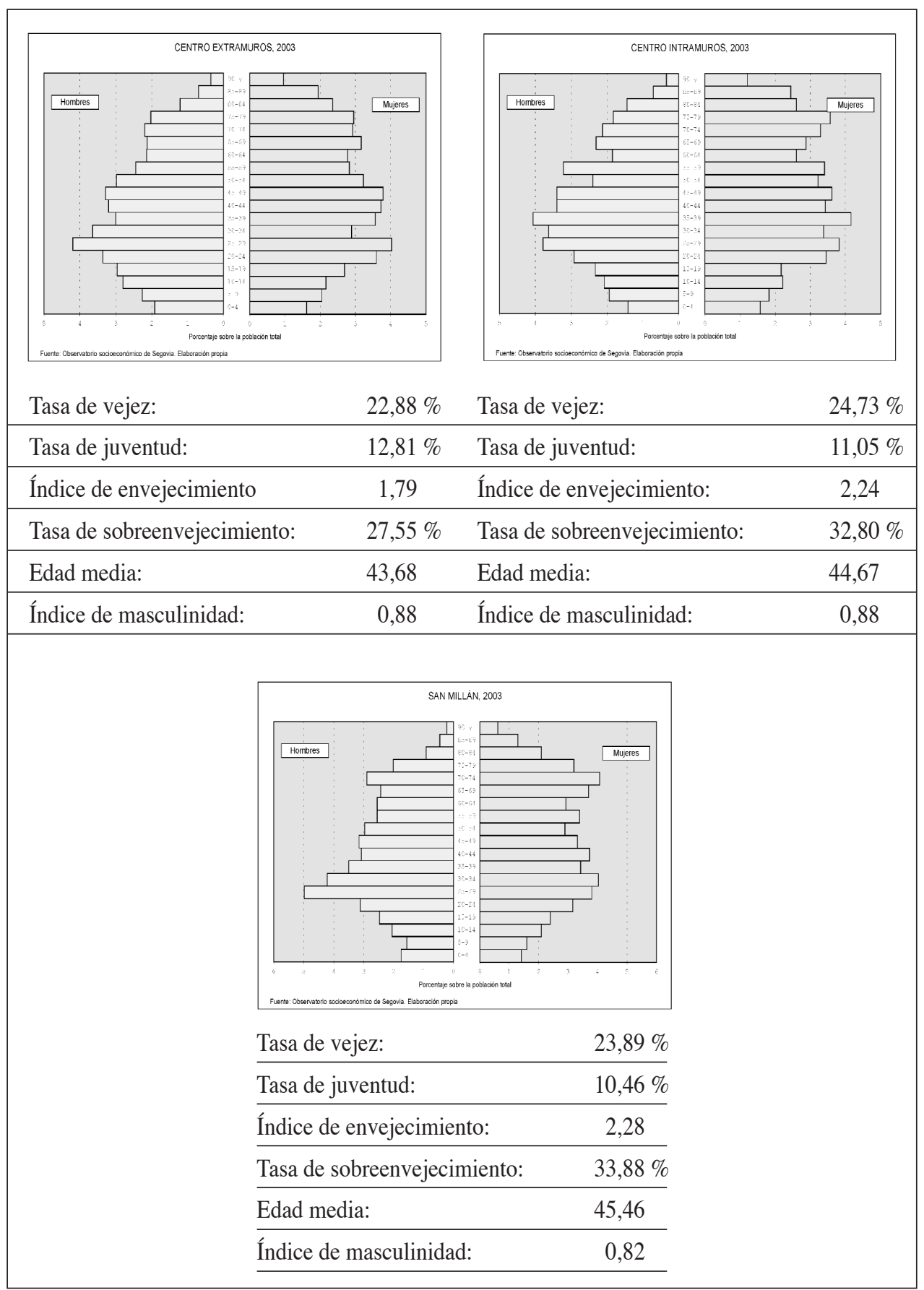

FIGURA 2. Pirámides de población e indicadores demográficos de la ciudad histórica. Fuente: O.S.S.: Datos socioeconómicos por barrios, 2003. 


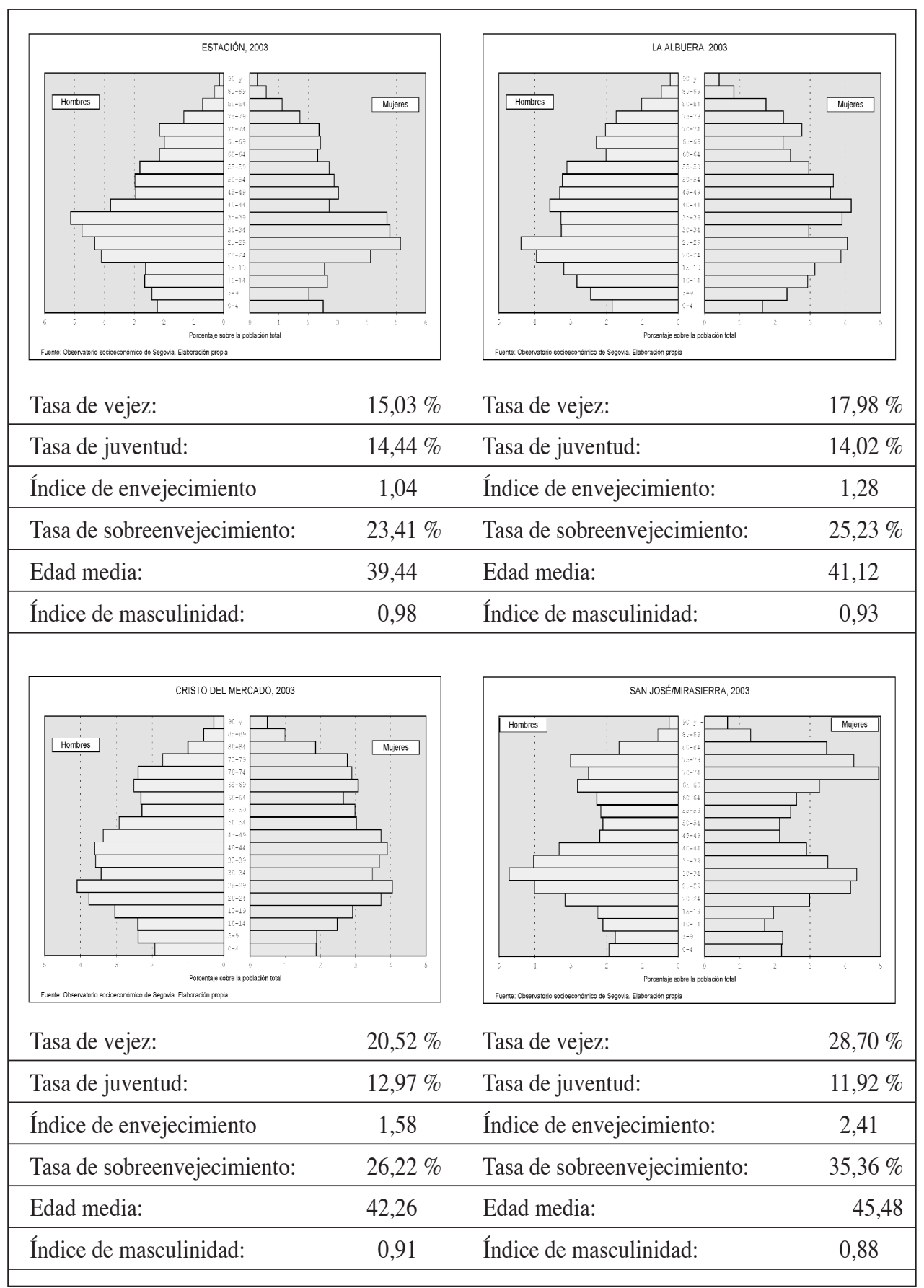

FIgura 3. Pirámides de población e indicadores demográficos de los barrios obreros. Fuente: O.S.S.: Datos socioeconómicos por barrios, 2003. 
confirmación del alto grado de envejecimiento y de sobreenvejecimiento de estos sectores «centrales» de la ciudad ${ }^{2}$.

Diferencias en definitiva, entre un centro histórico y unos barrios obreros que configuran el entramado urbano compacto, que va menguando sus vecindarios al tiempo que se van envejeciendo (además de feminizando), y unas áreas urbanas cada vez más alejadas, en continuo desarrollo, a las que van a vivir un gran número de parejas jóvenes, con hijos o en edad de comenzar a tenerlos. Así parece terminar de confirmarlo las desiguales edades medias de los residentes de uno y otro ámbito de la ciudad. De un lado, los espacios del crecimiento periférico, que no superan ninguno los 40 años de edad media (Nueva Segovia, 32,18, Estación, 39,11 y San Lorenzo, 39,44). En el extremo opuesto, los tradicionales y del «desarrollismo» (San José-Mirasierra, 45,48, Centro-Intramuros, 45,46, San Millán, 44,57, Centro-Extramuros, 43,68, Cristo del Mercado, 42,26 y La Albuera, 41,12), que los superan ampliamente.

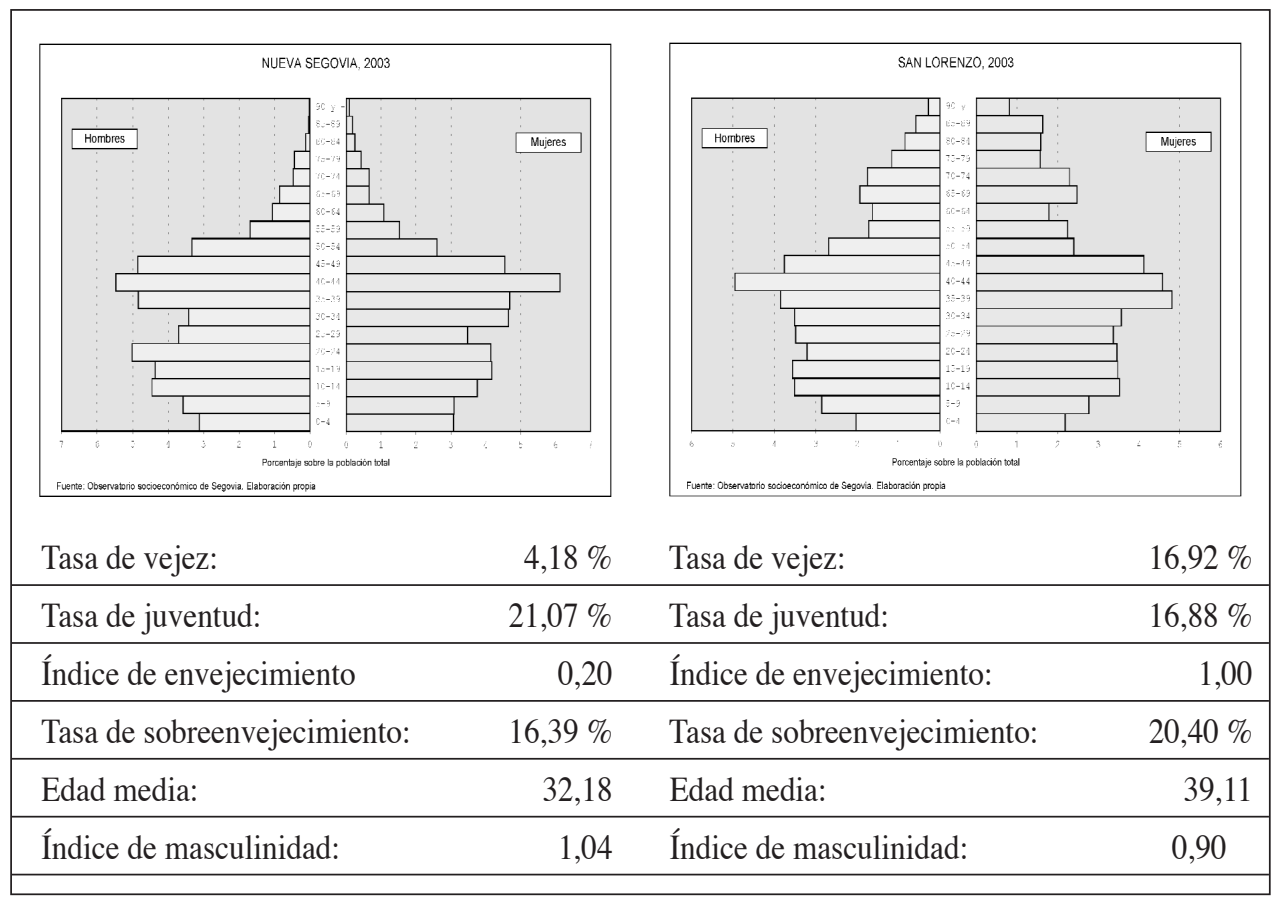

FiguRa 4. Pirámides de población e indicadores demográficos de la periferia urbana.

Fuente: O.S.S.: Datos socioeconómicos por barrios, 2003.

2 Unos desequilibrios muy acusados que se producen siempre a favor de las mujeres, dada la mayor esperanza de vida de que disfrutan. De ahí que el alto grado de envejecimiento y de sobreenvejecimiento (San Lorenzo) se identifique también con el carácter altamente feminizado de los colectivos vecinales residentes en estos sectores. Únicamente en Nueva Segovia el número de hombres es superior al de mujeres (1,04), siendo prácticamente el mismo en el caso de San Lorenzo (1) y Estación $(0,98)$. 


\section{Conclusiones}

A lo largo de más de cincuenta años, la transformación espacial de Segovia se ha producido al ritmo que han marcado las dinámicas poblacionales. Del pequeño núcleo originario, entorno al casco histórico (Intramuros y Extramuros) y a los arrabales de San Millán y, el más alejado de San Lorenzo, se pasó, sin solución de continuidad, a una ciudad que se expandía, más allá de los límites tradicionales, por una serie de barrios que iban surgiendo como consecuencia del desarrollo manufacturero. La Albuera, Cristo del Mercado, Estación y San José-Mirasierra conformaron, de consuno con el entramado urbano anterior, un espacio «polinuclear», que con el tiempo se fue densificando hasta constituir, finalizada la década de los 80 , una verdadera ciudad compacta.

La atonía demográfica que ha caracterizado a Segovia durante los años noventa, y hasta el momento actual, no ha significado una pérdida de los impulsos urbanísticos ni una merma en la capacidad de creación de ciudad. Aparece, con ella, un nuevo proceso de desarrollo urbano, sin apenas aumentos de población, cuyo soporte estratégico se fundamenta en la dialéctica existente entre el centro y la periferia.

Es así como, el crecimiento periférico de la ciudad, a través del polígono residencial de Nueva Segovia y, en menor medida, de las promociones de viviendas de San Lorenzo (o incluso de barrios obreros como Estación), además de algunas pequeñas urbanizaciones en los núcleos «incorporados», puede ser explicado por el paulatino y paralelo descenso del número de residentes en la ciudad compacta «central». Unas diferencias más que evidentes entre estos dos ámbitos que se traducen, también, en un desigual grado de envejecimiento, mucho más acusado en el primero de ellos.

\section{Fuentes}

I.N.E.: Censo de Población, 2001.

O.S.S. (Observatorio Socioeconómico de Segovia): Datos socioeconómicos por barrios, años 1996 y 2003.

\section{Bibliografía}

ÁLVAREZ MORA, A. (1999): «El uso del espacio en el centro histórico de Segovia en el marco de los programas de renovación y conservación de su contenido patrimonial». En CAMPESINO FERNÁNDEZ, A. (ed.): Comercio, turismo y cambios funcionales en las Ciudades españolas Patrimonio de la Humanidad. Cámara Oficial de Comercio e Industria de Cáceres. Cáceres, pp. 111-131.

CALDERÓN CALDERÓN, B. (2001): «Un espacio creado y transformado por el permanente conflicto entre centro y periferia: una hipótesis - más - para interpretar el modelo de crecimiento urbano en España». En MANERO, F. (coord.): Espacio Natural y Dinámicas Territoriales. Homenaje al Dr. D. Jesús García Fernández. Universidad de Valladolid. Valladolid, pp. 589-598.

GARCÍA BALLESTEROS, A. y BOSQUE SENDRA, J. (1989): El espacio subjetivo de Segovia. Universidad Complutense de Madrid. Madrid, 125 pp.

LUENGO GALLEGO, J. A. (2003): «El crecimiento urbano periférico en Segovia». Ería, $\mathrm{n}^{\circ}$ 61, pp. 197-208.

MARTÍNEZ DE PISÓN, E. (1976). Segovia. Evolución de un paisaje urbano. Colegio de Ingenieros de Caminos, Canales y Puertos. Madrid, 442 pp. 
SESMA, M. (1987): El nuevo espacio periférico de Segovia. Cuarenta años de teoría y práctica urbanísticas. Consejería de Educación y Cultura de la Junta de Castilla y León. Valladolid, $211 \mathrm{pp}$.

VILLAR CASTRO, J. (1989): «Estructura y tipología de las ciudades castellano-leonesas». En CABO, A. y MANERO, F. (dir.): Geografía de Castilla y León, T. 6. Ámbito. Valladolid, pp. 132-177.

VV. AA. (1994): Segovia, Ecología y Paisaje. Guía para una comprensión integral de la ciudad. Ayuntamiento de Segovia y otros. Segovia, 416 pp. 